Portland State University

PDXScholar

Engineering and Technology Management

Faculty Publications and Presentations

8-1-2015

\title{
Assessing Emerging Automotive Technologies for the Future
}

\author{
Aurobindh Kalathil Puthanpura \\ Portland State University \\ Rafaa Khalifa \\ Portland State University \\ Leong Chan \\ Portland State University
}

Follow this and additional works at: https://pdxscholar.library.pdx.edu/etm_fac

Part of the Operations Research, Systems Engineering and Industrial Engineering Commons Let us know how access to this document benefits you.

\section{Citation Details}

Puthanpura, A. K., Khalifa, R., \& Chan, L. (2015, August). Assessing emerging automotive technologies for the future. In Management of Engineering and Technology (PICMET), 2015 Portland International Conference on (pp. 2113-2120). IEEE.

This Article is brought to you for free and open access. It has been accepted for inclusion in Engineering and Technology Management Faculty Publications and Presentations by an authorized administrator of PDXScholar. Please contact us if we can make this document more accessible: pdxscholar@pdx.edu. 


\title{
Assessing Emerging Automotive Technologies for the Future
}

\author{
Aurobindh Kalathil Puthanpura, Rafaa Khalifa, Leong Chan \\ Department of Engineering and Technology Management, Portland State University, Portland, Oregon - USA
}

\begin{abstract}
A scenario-based multi-attribute decision-making (MADM) methodology has been developed and applied for the selection of automotive technology. The present study discusses, what are the problems for the current automotive technologies are, what requirements are outlined in the literatures and government guidelines/publications, considering different aspects of technological needs, public needs, policy measures etc. A set of criteria are developed, which cover multiple perspectives; reflecting the diverse stakeholders in the technology assessment, acquisition and adoption, in this case the government, the general public which includes drivers and pedestrians, automobile manufacturers. Three emerging technologies (Vehicle-to-Vehicle communication, Vehicle-toInfrastructure and Full autonomous) were identified and evaluated based on the mentioned criteria using Technique for Order of Preference by Similarity to Ideal Solution (TOPSIS), a multi criteria decision tool. Since the technologies are still being developed and some are barely emerging, it is considered appropriate to use speculative values from the publications from industry and other credible sources and consider multiple scenarios each of which could occur. In our case, we considered 9 main scenarios and evaluated the three technology candidates under each of them.
\end{abstract}

\section{INTRODUCTION}

Several researches in Intelligent Transportation system (ITS) assume that vehicles will be able to communicate speed and location data to roadway infrastructure and to other vehicles. Intelligent transport systems vary in technologies applied, ranging from management systems such as traffic signal control systems, car navigation, variable message signs, container management systems, automatic number plate recognition, or speed cameras to monitor, and to more advanced applications that integrate live data and feedback from a number of other sources, such as information systems, parking guidance, weather information, de-icing systems, and the like. U.S. Department of Transportation defined Intelligent Transportation Systems (ITS) in their final report, April 2011, as the application of advanced information and communications technology to surface transportation in order to achieve enhanced safety and mobility while reducing the environmental impact of transportation. The addition of wireless communication systems offers a powerful and transformative opportunity to establish transportation connectivity that further enables cooperative systems and dynamic data exchange using a broad range of advanced systems and technologies [1].

Significant developments in the ITS Program occurred in 2009 as the engineering research conducted under the Vehicle Infrastructure Integration (VII) program concluded and a program focused on deployment was started. The VII research program had been created in 2001 to study the potential of using dedicated short-range communications (DSRC) - both between vehicles and the roadway-to significantly improve road safety. VII proof-of-concept tested in 2007 demonstrated the viability of $5.9 \mathrm{GHz}$ DSRC-based safety applications in broad-spectrum, but left open many questions, including how DSRC technologies would find their way into vehicles and the transportation infrastructure. Today's ITS Program builds from the work under VII but with a number of important differences, as illustrated in Appendix table 1.

Vehicles have increasingly effective driver assistance and protection mechanisms. Various onboard controls and information sources allow the driver to customize her driving experience and remain up to date on the vehicle status. Passive safety mechanisms protect the passengers and the vehicle against adverse driving conditions (e.g., anti-lock braking systems (ABS)), navigation systems, compasses, rear and front parking radars, and cameras are the most common among autonomous sensor technologies. Recent technological developments, wireless communication, notably in mobile computing, and remote sensing, are now pushing intelligent transportation systems (ITS) toward a major leap forward. Vehicles are already integrated with sophisticated computing systems and onboard sensors each dedicated to one part of the cars operation. The new element is addition of new wireless communication, computing and sensing capabilities. Interconnected vehicles not only collect information about themselves and their environment, but they also exchange information in real time with other nearby (in principle) vehicles [2]. The development of vehicle communications is more active in Europe, USA, and Japan. In Europe E-safety research program from EU Intelligent Car Initiative, and industry driven project $\mathrm{V} 2 \mathrm{~V}$ Communication Consortium are some of the lead actors; In U.S, the V2I technology and the California Partners for Advanced Transit and Highways (PATH); In Japan, the Advanced Safety vehicle (ASV) Program, are notable and some testing has been conducted in this regard. Among the ASV technologies, adaptive cruise control (ACC), lane keeping support system, automatic braking system for reducing injury, curve overshooting preventing support system and night time forward pedestrian advisory system are now available in market. While some new technology ideas are under research and others are at driving test stage [3].

Considering the problems and development in the automotive industry we have developed and applied scenariobased multi-attribute decision-making (MADM) methodology for the selection of automotive technology. This would be the main focus of the paper and reminder of this paper is organized in 6 sections. Section 2 reviews the 
literature. Section 3 describes the methodology that have been used to conduct the study, candidate technology, data collection and the scenario analysis explanations. Section 4 details the empirical results of the analysis and followed by discussion of the results, conclusion and future research are outlined.

\section{LITERATURE REVIEW}

In the past 125 years since the first automobile was invented around 1885 [4], people have never stopped seeking new means to improve vehicles, traffic, transportation systems for efficiency, safety, environmental sustainability, and comfort [5]. Many efforts have been undertaken by vehicle manufactures to mitigate road accidents by focusing on both active and passive safety system. The main purpose of these efforts to achieve reasonable reduction of road deaths [6] [7] . This has primarily been achieved by application of communication technology along with vehicular environments such as anti-collision warning, probe data collections, intersection safety and traffic information [8]. Varied types of control systems and information sources provides driver with the opportunity to customize his/her driving experience and remain up to date on the vehicle status; passive safety mechanisms to protect the passengers and the vehicle against adverse driving conditions (e.g., antilock braking systems). Navigation systems, rear and front parking cameras, and radars are the most common among autonomous sensor technologies for the above said purpose [9]. Communication for Vehicle to Infrastructure (V2I): use vehicle and their sensors as sources for traffic control measurement. Communication for Infrastructure to Vehicle (I2V): provide vehicles and drivers information related to safety and infrastructure which are real-time, personalized, more accurate and reliable than that currently provided on VMS, radio, etc. [10]. One of important features of automotive technologies is safety. Safety applications minimize the risk of accidents and, most important, reduce the severity of the accident if it still occurs (incident management, collision avoidance, etc.) [11]. In emergency situations, a driver typically relies on the other car driver reaction that shows by the tail brake light of the car immediately ahead to decide his or her own braking action. Under typical road conditions, this is not always the best collision avoidance strategy for various reasons [12]. Furthermore, as noticed and reported in some previous research, in many cases, the ability to detect emergency event occurring at some distance ahead is limited by the inability of drivers to see past the vehicle in front of them. Also, fuel efficiency features as one of important factors that leads to development and improvement of automotive technology. Under the Energy policy conservation Act (EPCA) which established mandatory fuel economy standards for all new automobiles sold in the United States [13]. Moreover, called the Corporate Average Fuel Economy (CAFE) were designed to increase the incentive for automotive producers to improve fuel efficiency beyond that dictated by market forces. It also indicated that all automobile producers with sales in the U.S. market were enforced to comply under the CAFE program; they must meet a minimum average fuel-efficiency standard [13]. The cost penalty is especially substantial for the low- to moderate-content vehicles that have dominated most automobile manufacturers' recent production volumes. This cost trade-off is one of the key components of standard financial analysis used to evaluate new technologies in the industry [14]. The cost of advanced technology components required for automation is greater for higher content levels.

Autonomous technologies have been developed over several decades to support human drivers. Some of these technologies include anti-lock brake system, traction and cruise control, warning systems, parking assist - to provide some level of autonomy. The Rand corporation report on autonomous vehicles research indicates that these systems have indeed helped to reduce road crushes to a very great extent. However, all these still require the driver to provide a level of control and monitoring whilst on the road in order to stay safe - which means regardless of the kind of new features which are added to assist the driver, his decision is very much required during driving [15].

Even with some of the advancement made in developing safety technologies in cars, public records for the United States shows that in 2011, 39\% of road fatalities has something to do with alcohol [15] [16]. This trend shows that the existing advancements still essentially are not close to fixing the road crash fatalities issues due to alcohol.

With today's automotive intelligence technologies, the driver is the one at helm of affairs, including which road to destination, where to get parking spot and at what speed to accelerate on a highway. Since the driver might be wrong in his judgment in all these decisions, it is apparent that fuel usage is not efficient since the driver might not know which roads are busy, or which parking lot is available. Research has it that several gallons of fuel are wasted everyday looking for parking lot [16]. Again, there is evidence suggesting that driving practices of uniform acceleration helps to efficiently burn fuel; the question to answer is, "Does the current level of vehicle automation ensure the optimal consumption of fuel?" [15]. Thirdly, the past and present vehicles incorporate in them heavy metals to keep them stable for safety reasons. If we can develop automobiles which can ensure enhanced safety using intelligent sensors or other connected communication technologies, $20 \%$ of weight decrease corresponds to $20 \%$ fuel efficiency [16].The inefficient use of fuel leads to greenhouse gases which has a tremendous cost implications in terms of threat of this planet and associated cost to beat these emission down; for instance the Environmental protection agency (EPA) estimates the annual cost of greenhouse gases for the US alone is about $\$ 41$ billion [15].

In the US road congestion leads to a cost of about 4.8 billion hours of time, 1.9 billion gallons of wasted fuel (equivalent to two months 'operation of the Alaska Pipeline), 


\section{Proceedings of PICMET '15: Management of the Technology Age}

and $\$ 101$ billion in combined delay and fuel costs, aside cost associated with travel time and dependability. These kinds of loses are still very worrying trends, which impact our lives [17].

The tasks associated with operating a vehicle that requires effortful processing can drain a driver's cognitive resources, leading to the stressing of the driver. In fact, considering all aspects of the driving task, one might argue the complexity of operating a modern automobile has outstripped the capability of the human nervous system. But as long as there is still the action of the driver and sometimes for long distances it is still stressful and is a contributing factor to several road accidents [18].

It is estimated that at least one out of four North American and European citizen will be over 65 by 2020 and might have some kind of disability which can impact their driving capabilities. The existing autonomous car technologies do not take into consideration the aging population and the disabled [19].

\section{METHODOLOGY}

Multiple-attribute Decision-making methodology is the process of finding the best option from all of the possible alternatives. In almost all such complications the multiplicity of criteria for judging the alternatives is pervasive. That is, for many such problems, the decision maker wants to solve multiple criteria decision making (MCDM) problem [20]. One such methodology is Technique for Order Preference by Similarity to Ideal Solution (TOPSIS). TOPSIS is used to rank the best alternative from three different alternatives. The methodology is extended to include scenario analysis to mimic real life situations. Since information available to decision makers may vary in quality and scale, it becomes challenging to make real life decisions.

TOPSIS is a simple ranking method in conception and application developed by Hwang and Yoon in 1981. It is an extension of theory of ideal solutions developed by Zeleny [21] in 1974. The basic principle is governs that the chosen alternative should have the shortest distance from the ideal solution and the farthest distance from the negative-ideal solution [22]. The positive ideal solution maximizes the benefit criteria and minimizes the cost criteria, whereas the negative ideal solution maximizes the cost criteria and minimizes the benefit criteria. TOPSIS is a technique that combines quantitative attribute (such as price, distance, time, and so on) and qualitative attributes (such as quality of relationship, quality of assurance, reliability) and compares all alternatives together based on these attributes [23]. TOPSIS also provides cardinal ranking of alternatives, making apt use of attribute information, and does not require attribute preferences to be independent.

\section{A. Technique for Order Preference by Similarity to Ideal Solution (TOPSIS) \\ TOPSIS methodology is created using a series of steps}

described below [24].

Step 1: Calculate the normalized ratings

Normalizing the attribute value information as it is available in different scales. The normalization converts dimension attributes to non-dimension attributes allowing to compare across attributes.

$$
r_{i j}=\frac{x_{i j}}{\sqrt{\sum_{i=1}^{m} x_{i j}^{2}}}
$$

Where $r_{i j}$, is the normalized score matrix and $x_{i j}$ is the score of the $\mathrm{j}^{\text {th }}$ indicator for $\mathrm{i}^{\text {th }}$ alternative and there are $\mathrm{n}$ attributes and $\mathrm{m}$ alternatives.

Step 2: Calculate the weighted normalized ratings

The weighted normalized matrix is calculated as

\[ a_{i j=w_{j}} r_{i j} \]
Where $\mathrm{w}_{\mathrm{j}}$ is the weight of the $\mathrm{j}^{\text {th }}$ attribute.

Step 3: Identification of Positive Ideal and Negative Ideal solution

Positive ideal solution (PIS) is found by finding the maximum $a_{i j}$ value for each set of benefit attribute and minimum $a_{i j}$ value for each set of cost attribute whereas Negative ideal solution (NIS) is found by finding the minimum $a_{i j}$ value for each set of benefit attribute and maximum $\mathrm{a}_{\mathrm{ij}}$ value for each set of cost attribute.

$$
\begin{aligned}
& P I S=\left\{\left(\max a_{i j} \mid j \in j_{1}\right),\left(\min a_{i j} \mid j \in j_{2}\right) \mid i=1, \ldots, m\right\} \\
& N I S=\left\{\left(\min a_{i j} \mid j \in j_{1}\right),\left(\max a_{i j} \mid j \in j_{2}\right) \mid i=1, \ldots, m\right\}
\end{aligned}
$$

Where $\mathrm{j}_{1}$ is set of benefit attributes, $\mathrm{j}_{2}$ is set of cost attributes, and $\mathrm{j}_{1}+\mathrm{j}_{2}=\mathrm{n}$ (total number of attributes).

\section{Step 4: Calculate separation Measures}

The separation measure between attributes is measured by the n-dimensional Euclidean distance. The separation of each alternative from the positive ideal solutions, $\mathrm{D}^{+}$, is given by the formula:

$$
D_{i}^{+}=\sqrt{\sum_{j=1}^{n}\left(a_{i j-} a_{j}^{+}\right)^{2}, i=1, \ldots, m}
$$

The separation from the negative ideal solutions, $\mathrm{D}^{-}$is given by the formula:

$$
D_{i}^{-}=\sqrt{\sum_{j=1}^{n}\left(a_{i j-} a_{j}^{-}\right)^{2}, i=1, \ldots, m}
$$

Step 5: Calculate similarities to Positive Ideal solution

Similarities to Positive ideal solution are calculated using the formula:

$$
R_{i}^{*}=\frac{D_{i}^{-}}{\left(D_{i}^{+}+D_{i}^{-}\right)}, \mathrm{i}=1, \ldots, \mathrm{m}
$$


Note $0 \leq R_{i}^{*} \leq 1$, where $R_{i}^{*}=0$ when $\mathrm{D}_{\mathrm{i}}=\mathrm{D}^{-}$, and $R_{i}^{*}=1$ when $\mathrm{D}_{\mathrm{i}}=\mathrm{D}^{+}$

\section{B. Candidate Technology}

\section{Vehicle to Vehicle (V2V)}

Vehicle to Vehicle (V2V) is an automotive technology that is designed to allow automobiles to communicate with each other. V2V system uses dedicated short range communication (DSRC) which is in the $5.9 \mathrm{GHz}$ frequency. This frequency is also used by U-NII devices (Unlicensed National Information Infrastructure).

Federal Communications Commission (FCC) has allocated spectrum for use by DSRC technologies that are part of DOT's ITS research program. Allocation involves segmenting spectrum used for wireless communication into bands of frequencies that are allocated for use by particular types of services. FCC manages spectrum use for non-federal users, including private, commercial, and state and local government users; the Department of Commerce's National Telecommunications and Information Administration manages spectrum for federal users (47 U.S.C. $\S \S 303,305$ ) [25] [25]. Specifically, in 1999, FCC allocated 75 megahertz $(\mathrm{MHz})$ of spectrum $^{1}$ - the 5.850 to 5.925 gigahertz $(\mathrm{GHz})$ band (5.9 GHz band) — for the primary purpose of improving transportation safety and adopted basic technical rules for DSRC operations ${ }^{2}$. In 2003, FCC established licensing and service rules for the $5.9 \mathrm{GHz}$ band to provide short-range, wireless link for transferring information between vehicles and roadside systems ${ }^{3}$. However, the President and Congress have responded to growing demand for wireless broadband services by making changes in the law to promote efficient use of spectrum, including the bands previously set aside for use by DSRC-based technologies.

The communication technology will enable vehicles to exchange vital information 10 times per second, about location, acceleration, speed, and braking. Cars will be able to calculate the hazard risks within about 300 meters and alert their drivers or even take automatic collision-avoidance action [26].

\footnotetext{
${ }^{1}$ In the Matter of Amendment of Parts 2 and 90 of the Commission's Rules to Allocate the 5.850-5.925 GHz Band to the Mobile Service for Dedicated Short Range Communications of Intelligent Transportation Services, Report and Order, 14 FCC Rcd 18221 (1999).

${ }^{2}$ Radio frequencies are grouped into bands and are measured in units of Hertz, or cycles per second. The term megahertz $(\mathrm{MHz})$ refers to millions of Hertz and gigahertz $(\mathrm{GHz})$ to billions of Hertz. The Hertz unit of measurement is used to refer to both the quantity of spectrum (such as 75 $\mathrm{MHz}$ of spectrum) and the frequency bands (such as the $5.850-5.925 \mathrm{GHz}$ band).

${ }^{3}$ Amendment of the Commission's Rules Regarding Dedicated Short-Range Communication Services in the 5.850-5.925 GHz Band (5.9 GHz Band); Amendment of Parts 2 and 90 of the Commission's Rules to Allocate the 5.850-5.925 GHz Band to Mobile Service for Dedicated Short Range Communications of Intelligent Transportation Services; WT Docket No. 0190, ET Docket No. 98-95, Report and Order, 19 FCC Rcd 2458 (2004) (FCC 03-324).
}

\section{Vehicle to Infrastructure (V2I)}

V2I is defined as a communication and cooperative exchange of data between vehicles via wireless technologies, within a range that can vary from a few meters to a few hundred meters. In order, to improve road safety, quality, efficiency and when-necessary increase the capacity of the road. This technology has been conceived to deal with such as a small time interval: even through an accident has occurred at a distance which would make it difficult for the driver to react promptly, the information is rapidly transmitted to the vehicle and if required an automatic system intervenes, possibly without the driver's involvement, to prevent the accident or other consequential accidents [27].

The V2I computing, communication, and sensing equipment and user interfaces will be, in most cases, new with respect to the current on-board equipment. In terms of sensing and user interface hardware and software, V2I technology and its systems will leverage on the array of equipment vehicles currently carry; for example, data concerning the vehicle operation will be obtained via the corresponding or upgraded onboard interfaces. In general, V2I technology will not be developed from scratch; rather, as ongoing projects show, mature and well understood components and their variants will be the basis [9].

The new objectives of transport systems and mobility concern: quality, efficiency, safety and security. One of the most interesting tools related to safety refers to a set of applications that involve interaction and cooperation between an infrastructure and in-vehicle systems.

\section{Autonomous Vehicles}

Over the past 50 years, there has been several innovations in the automotive industry to create autonomous technologies to provide some level of assistance to human drivers [28]. Examples of such technologies include advanced cruise control, anti-lock brake systems, collision avoidance systems and several others which companies have incorporated on incremental basis to provide some level of control and support to drivers. Our definition of autonomous cars is beyond the incorporation of some level of autonomous behavior in a car- Autonomous cars for our purpose represent a leap from providing support systems to human drivers to developing self-driving cars, which require no human control to move from point $\mathrm{A}$ to $\mathrm{B}$ [28] [15].

Autonomous cars offers the possibility of fundamentally changing transportation, and promises to do away with several kinds of interactions with cars which required human cognition, control and monitoring [16]. Levels of autonomy as illustrated in Appendix table $\mathbf{2}$ are regarded as the highest level of classification established by the National Highway Traffic Safety Administration. The benefits which can be derived depends largely on the level of autonomy provided, for example a car with anti-lock brake system will only provide the safety associated with such a technology whereas one with full autonomy as defined above has all conceivable safety and other kinds of benefits. 


\section{Proceedings of PICMET '15: Management of the Technology Age}

Unlike existing cars (level 0 through 3) where the level of autonomy still requires the human driver to be aware of his environment and driving conditions in order to make accurate decisions, the autonomous car will be capable of sensing its environment and navigating without human input [28].

\section{Data collection}

Data collection was done though survey with an expert in the field. The attributes were rated on 7 point Likert scale 1 being the lowest and 7 being the highest. The attributes that were rated were grouped into two categories benefit attributes and cost attributes. Safety, fuel efficiency, compatibility, availability were grouped as benefit attributes and cost is grouped as cost attributes. The attributes were selected based on literature review as illustrated in Appendix Table 3. The values for the data collect is illustrated in appendix Table 4. Availability attribute is calculated based on differences between current year (2014) and when the technology will be available. V2V technology is expected to be in the market by 2018 so the value is (2014-2018) -4. V2I technology is expected to be in the market soon after that as that is an extension of V2V technology so the value is (2014-2021) -7. Autonomous vehicle technology is expected by 2025 so the value is $(2014-2025)-11$.

\section{Scenarios}

In order to mimic the real world situation nine scenarios and no scenario were created and based on the scenarios each attribute was assigned weights. In Appendix Table 5, the scenarios and the weights are elaborated upon. This is performed to create the scenarios push-pull framework to policy decisions and influence the supply of new knowledge directly. There are two ways governments can encourage innovation: technology-push, implement measures to reduce the public measures and demand-pull, implement measures that increases the private payoff to successful innovation [29]. Three different policy instruments were used to create the six different scenarios in three different time frames. The three policy instruments are government sponsored R\&D, tax credit for companies to invest in $R \& D$, and tax credit and rebates for consumers for adoption of new technology. The three time frames used for the study are short term, medium term, and long term.

No Scenario depicts non real solution. Scenario 1, scenario 2 and scenario 3 assess the impact of the government sponsored R\&D in the three different time frames. Scenario 1 depicts the situation where the initial government sponsored R\&D is assessed in the short term time frame. Even with the policy in place the cost of the technology is high as it's a new technology and as the technology evolves the cost of the technology will lower. Scenario 2 and scenario 3 depict the situation in medium and long term with the cost of the technology decreasing. Scenario 4, scenario 5, and scenario 6 assess the impact of the tax credit for companies to invest in R\&D in the three different time frames. Scenario 4 depicts the situation where the companies are given tax credit to invest in $\mathrm{R} \& \mathrm{D}$ in the short term that lowers the cost of innovation in the initial stages. However, as the tax credit policy diminishes the cost of the investment will increase in the scenario 5 and 6 as the time frames increases. But the knowledge gained in the initial stages will reduce the cost in the long run as they have a knowledge of the technology. Scenario 7, scenario 8, and scenario 9 assess the impact of the tax credit and rebates for consumers in the three different time frames. Scenario 7 depicts the situation where the consumers are given rebate for the adoption of this technology in the short term the cost of the technology will be higher. However, as time progresses as depicted in scenario 8 and 9 and with improvement of the technology the cost to the technology will gradually decrease and will be competitive with other traditional vehicles in the market.

\section{RESULTS AND DISCUSSIONS}

Table 6 provides detail results of all the scenarios and time frame discussed above. For the no scenario case all the attributes are given equal weights. Even in this case V2V (with a score of 0.883) is ranked as the most preferred alternatives since the attribute rated for $\mathrm{V} 2 \mathrm{~V}$ are higher. $\mathrm{V} 2 \mathrm{~V}$ is ranked as the most preferred alternative (with a score of 0.883), while V2I holds the second rank (with a score of 0.576 ) for scenario 1. This outcome is reasonable for Scenario 1 as it depicts a situation in which alternatives are evaluated in the short term and with Government sponsored R\&D. V2V has lower cost than other alternatives and satisfactory performance in terms of safety and compatibility. Scenario 2 and Scenario 3 are similar to Scenario 1 with only difference being the time frame which would help consumers with more options as there are more competitors in the market that would drive the price down.

Scenario 4, Scenario 5, and Scenario 6 depicts the situation in which the alternatives are evaluated where companies are provided Tax credit to invest in R\&D. V2V is ranked as the most preferred alternatives (with a score of 0.923 ) followed by V2I (with a score of 0.573 ) in scenario 4. Scenario 5 and Scenario 6 are similar to Scenario 4 only difference being the time frame still V2V is the most preferred alternative (with a score of $0.915,0.900$ ) followed by V2I (with a score of $0.552,0.522$ ). This is expected since in the medium term and long term the technologies are in use and the cost of the alternatives is lower than their initial price.

Scenario 7, Scenario 8, and Scenario 9 depicts the situation in which the alternatives are evaluated in which Tax credit and rebates for consumers are given for technology adopters. $\mathrm{V} 2 \mathrm{~V}$ is ranked as the most preferred alternatives (with a score 0.920 ) followed by V2I (with a score of 0.563 ). Scenario 8 and Scenario 9 are similar to Scenario 7 only difference being the time frame still V2V is the most preferred alternative (with a score of $0.912,0.899$ ) followed by V2I (with a score of $0.540,0.499$ ). This is expected since autonomous vehicles are still expensive than other technologies. 
The result show that V2V will be most likely fit the safety needs of road transport in the short, medium and long terms whilst still balancing all the other criteria(Compatibility, Cost, Fuel Efficiency) and could be available sooner than the other candidates, whether or not there is policy to push it. More R\&D to speed up V2V implementation will help provide more safety features for existing cars and that same technology can further speed up the development of full Autonomous cars.

The weight assignment for each of the scenarios are given based on cardinal scale of $0-100(0$ as the worst and 100 as the best for benefit criteria and vice -versa for cost criteria). This is used to transform qualitative criteria to quantitative one. Safety attribute was given a weight of 80 for all the scenarios. Since for successful adoption of these vehicular technologies it is mandatory for it to have the highest safety rating and any small error in the technology could lead to a devastating outcome. Fuel efficiency was given a weight of 50 for all the scenarios, because it would be dependent on the consumer to choose appropriate vehicles. Some consumer may weigh this attribute higher for they would prefer green vehicles and some would prefer vehicles with higher horse power, such as truck, for their towing capacity. Compatibility criteria was given weight of 80 for scenarios 1,4 , and 7 as they are related to short term. In the short term, it is important to know if these technologies are compatible with the existing ecosystem. With increasing adoption rate of these technologies consumer would be aware that these technologies are already compatible with the ecosystem as more new automobile replace older automobiles. The weight for medium term (scenarios 2, 5, and 8) and long term (scenarios 3, 6, and 9) are given in decreasing scale 70 - 50, as more adoption compatibility will not be of major concern for consumers. Availability criteria depicts the same condition as compatibility since as the time progresses the availability of the technology increases and improvement are also made due to additional knowledge gained through experience. Cost criteria for scenarios 3,6, and 9 are given lower score $(70-50)$ than other scenarios, since in the long term with improved adoption, the cost of the technology will be lower as the manufacturing process will improve. In the short term $(1,4$, and 7$)$ and medium term $(2,5$, and 8$)$ the cost would be initially high. However, with time and improvement in manufacturing process, acquired through the knowledge gained, the cost would go down.

\section{CONCLUSION}

The problem of selecting an appropriate vehicular technology alternatives is addressed in this study with a scenario-based MDM method. Nine scenarios are articulated, depicting the most commonly encountered decision situations and addressing the technology options. The methodology developed in this work effectively captures different policy options and the time frames in terms of weights for criteria for each scenarios and translates them into the mathematical algorithms of the MDMA methodology. Five attributes are used for evaluating alternatives that represent the characteristics of appropriate technologies for each scenario. The methodology developed efficiently identifies the appropriate technology for each scenarios.

For the no scenario case equal weights were assigned for the attributes, it is difficult to identify the most appropriate vehicular technology alternative. For the scenarios considered in the study, the alternatives are ranked according to the policy and the time frames. It should also be noted that it is not possible to achieve the optimal solution for each scenarios because there are a finite number of alternatives available. Therefore, the best available solution has to be selected. TOPSIS mimics the nature of this type of decisionmaking problem and is found to the efficient in identifying the best alternative for each of the scenarios.

\section{FUTURE RESEARCH}

The present study uses TOPSIS method to identify the best alternative. Future research would include Delphi method to include panel of experts to provide score for the attributes. We would also expand the attributes used for the TOPSIS method by surveying the population in order to get a better understating of the requirement for these technologies that users of these technologies value the most.

\section{ACKNOWLEDGEMENTS}

I am grateful for the help I received from Felix Sie during the initial research phase of this project.

\section{REFERENCES}

[1] S. Sill, B. Christie, A. Diephaus, D. Garretson, K. Sullivan and S. Sloan, "Intelligent Transportation Systems (ITS) Standards Program Strategic Plan for," April 2011. [Online]. Available: http://www.its.dot.gov/standards_strategic_plan/stds_strat_plan.pdf.

[2] P. Papadimitratos, A. La Fortelle, K. Evenssen, R. Brignolo and S. Cosenza, "Vehicular communication systems: Enabling technologies, applications, and future outlook on intelligent transportation," Communications Magazine, IEEE, pp. 84 - 95, 2009.

[3] A. Furda and L. Vlacic, "Enabling Safe Autonomous Driving in RealWorld City Traffic Using Multiple Criteria Decision Making," Intelligent Transportation Systems Magazine, IEEE, pp. 4-17, 2011.

[4] Traffic Safety Facts, National Center for Statistics and Analysis, 2005.

[5] F. Qu, F. Wang and L. Yang, "Intelligent transportation spaces: vehicles, traffic, communications, and beyond," communications Magazine, IEEE, 2010 . [Online]. Available: ieeexplore.ieee.org.

[6] F. M, F. M. P. Garrido, C. J, C. C and M. P, "Assistance through communication technologies and vehicles," IEEE, September 2012.

[7] C. MC and R. N, Assessing the impact of V2V/V2I communication systems on traffic congestion and emissions, Human Centred Design for Intelligent, 2010.

[8] C. W, H. K, C. H and O. H, Realization of anti-collosion warning application using V2V communication, IEEE, 2009.

[9] P. P and A. L. Fortelle, Vehicular communication systems: Enabling technologies, applications, and future outlook on intelligent transportation, IEEE, 2009.

[10] T. G and B. J, Comparative synthesis of the 3 main European projects 
dealing with Cooperative Systems (CVIS, SAFESPOT and COOPERS) and description of COOPERS, IEEE, 2008.

[11] D. K, B. M, J. Gaber and W. M, Wireless communication technologies for ITS applications [Topics in Automotive Networking], communications, IEEE, 2010.

[12] S. Biswas, T. R and D. F, Vehicle-to-vehicle wireless communication protocols for enhancing highway traffic safety, Communications Magazine, IEEE, 2006.

[13] C. R and G. J, "The effect of fuel economy standards on automobile safety," Journal of Law and Economics, 1989.

[14] M. L and D. R, "Integrating product mix and technology adoption decisions: a portfolio approach for evaluating advanced technologies in the automobile industry," Journal of Operations management, 2001.

[15] M. A. J, K. N, D. S. K, S. P, S. C and A. O. O, "Autonomous Vehicle Technology: A Guide for Policymakers," 2014. [Online]. Available: http://www.rand.org/pubs/research_reports/RR443-1.html.. [Accessed 2014].

[16] "Self-driving cars: The next revolution," KPMG, 2012.

[17] M. E. Hallenbeck, J. M. Ishimaru and J. Nee, "MEASUREMENT OF RECURRING VERSUS NON-RECURRING CONGESTION," 2014. [Online]. Available: http://www.wsdot.wa.gov/research/reports/fullreports/568.2.pdf. [Accessed 2014].

[18] N. D. Cottrella and B. K. Bartona, "The role of automation in reducing stress and negative affect while driving," Theoretical Issues in Ergonomics Science, pp. 53 - 68, 2011.

[19] S. Rosenbloom and A. Ståhl, "The Convergence of Environmental, Safety, Mobility and Community Design Issues," European Journal of Transport and Infrastructure Research, pp. 197 - 213, 2003.

[20] C.-T. Chen, "Extensions of the TOPSIS for group decision-making under fuzzy environment," Fuzzy Sets and Systems, pp. 1-9, 2000.

[21] M. Zeleny, "A concept of compromise solutions and the method of the displaced ideal," Computer \& Operations, pp. 479-496, 1974.

[22] S. Opricovic and G.-H. Tzeng, "Compromise solution by MCDM methods: A comparative analysis of VIKOR and TOPSIS," European Journal of Operational Research, pp. 445-455, 2004.
[23] A. T. Eshlaghy and M. Kalantary, "Supplier Selection by NeoTOPSIS," Applied Mathematical Sciences Vol. 5, 2011, no. 17, pp. 837 - 844, 2011.

[24] P. P. Kalbar, S. Karmakar and S. R. Asolekar, "Selection of an appropriate wastewater treatment technology: A scenario-based multiple-attribute decision-making approach," Journal of Environmental Management, pp. 158-169, 2012.

[25] "COMMERCIAL SPECTRUM: Plans and Actions to Meet Future Needs, Including Continued Use of Auctions," November 2011. [Online]. Available: http://www.gao.gov/assets/590/587319.pdf.

[26] G. Putic, "Vehicles May soon be Talking to Each other," 4 April 2014. [Online]. Available: http://www.voanews.com/content/vehicles-maysoon-be-talking-to-each-other-/1886895.html. [Accessed 2014].

[27] B. Dalla, F. Deflorio and S. Diwan, " Assessing the effects of intervehicle communication systems on road safety. Intelligent Transport Systems IET," 2009. [Online]. Available: www.ietdl.org.

[28] U. m. O. zgu" ner, C. Stiller and K. Redmill, "Systems for Safety and Autonomous Behavior in Cars:The DARPA Grand Challenge Experience," 2007.

[29] G. F. Nemet, "Demand-pull, technology-push, and goverment-led incentives for non-incremental technical change," Research Policy, vol. 38, pp. $700-709,2009$.

[30] S. Kraus, M. Althoff, B. HeiBing and M. Buss, "Cognition and emotion in autonomous cars," Intelligent Vehicles Symposium, 2009 IEEE, Xi'an, 2009.

[31] W. J. Mitchell. [Online]. Available: http://h20.media.mit.edu/pdfs/wjm2007-0509.pdf.

[32] "A Heritage of Inovation," Volvo cars, 2014. [Online]. Available: www.volvocars.com/us/about/our-company/heritage/innovations.. [Accessed 2014].

[33] L. H. A, "Decision Making for Technology Execuitves: Using Multiple Perspectives to Improve Performance,", Boston, MA, Artech House, 1999.

[34] W. Lindsay , "shrink that foot print," February 2013. [Online]. Available: $\quad \mathrm{http}: / /$ shrinkthatfootprint.com/wpcontent/uploads/2013/02/Shades-of-Green-Full-Report.pdf. [Accessed 2014].

\section{APPENDIX}

\begin{tabular}{|c|c|c|}
\hline Attribute & VII Engineering Research & ITS Program Focused Toward Deployment \\
\hline Communications technologies & DSRC only & $\begin{array}{l}\text { Best technology for intended application } \\
\text { (DSRC for safety) }\end{array}$ \\
\hline In-vehicle devices & OEM production units only & Aftermarket and retrofit opportunities \\
\hline Vehicle focus & Light vehicles & All vehicle types \\
\hline Stakeholder involvement & Limited & Broad engagement \\
\hline International focus & Limited & Significant international harmonization effort \\
\hline Program cohesion & Loosely coupled research programs & $\begin{array}{l}\text { Strong, collective USDOT support, } \\
\text { coordination, and leadership }\end{array}$ \\
\hline Deployment focus & $\begin{array}{l}\text { Limited - oriented toward prototyping and } \\
\text { proof of concept - See more }\end{array}$ & Strong deployment focus \\
\hline
\end{tabular}

Source: U.S. Department of Transportation

TABLE 2: LEVEL OF AUTOMATION

\begin{tabular}{|l|l|}
\hline Level of Automation & Description \\
\hline Level 0 & Human driver completely in control of all functions \\
\hline Level 1 & One function is automated \\
\hline Level 2 & More than one function automated at the same time \\
\hline Level 3 & $\begin{array}{l}\text { The driving functions are sufficiently. Driver can safely do other } \\
\text { things }\end{array}$ \\
\hline Level 4 & The car drives itself without human input \\
\hline
\end{tabular}


TABLE 3: ATTRIBUTES

K. Dar, M. Bakhouya, J. Gaber, M. Wack. 2010 [11]

S. Biswas, R. Tatchikou, F. Dion, 2006. [12]

R. Crandall, J. Graham. 1989 [13]

L. Morgan, R. Daniels, 2001 [14]

I. Hsu, M. Wódczak, R. White, 2010

U. m. O. zgu"ner, C. Stiller and K. Redmill, 2007. [28]

J. M. Anderson, N. Kalra, K. D. Stanley, P. Sorensen, C. Samaras and O. A. Oluwatola, $\mathbf{X}$ 2014 [15]

KPMG, "Self-driving cars: The next revolution," 2012. [16]

S. Kraus, M. Althoff, B. HeiBing and M. Buss, 2009. [30]

"Autonomous Cars - Not if, but when," Automotive Technology Research, 2014.

W. J. Mitchell, 2007 [31]

Volvo Cars, 2014 [32]

Boston, MA, Artech House, 1999. [33]

Lindsay Wilson, 2013 [34]

\begin{tabular}{|l|l|l|l|l|}
\hline Safety & $\begin{array}{l}\text { Fuel } \\
\text { efficiency }\end{array}$ & Cost & Availability & Compatibility \\
\hline $\mathbf{X}$ & $\mathbf{X}$ & & & \\
\hline $\mathbf{X}$ & & & & \\
\hline & $\mathbf{X}$ & $\mathbf{X}$ & & \\
\hline $\mathbf{X}$ & & & & \\
\hline $\mathbf{X}$ & & $\mathbf{X}$ & $\mathbf{X}$ & \\
\hline $\mathbf{X}$ & $\mathbf{X}$ & $\mathbf{X}$ & & $\mathbf{X}$ \\
\hline & & $\mathbf{X}$ & & $\mathbf{X}$ \\
\hline & & & & $\mathbf{X}$ \\
\hline $\mathbf{X}$ & & & & $\mathbf{X}$ \\
\hline & & & & \\
\hline
\end{tabular}

TABLE 4: VALUE ASSIGNMENTS FOR ATTRIBUTES

\begin{tabular}{|c|c|c|c|c|c|}
\hline & \multicolumn{4}{|c|}{ Benefit Attribute } & \multirow{2}{*}{$\begin{array}{l}\text { Cost Attribute } \\
\text { Cost }\end{array}$} \\
\hline & Safety & Fuel efficiency & Compatibility & Availability & \\
\hline V2V & 7 & 5 & 7 & -4 & 3 \\
\hline V2I & 4 & 6 & 7 & -7 & 4 \\
\hline $\begin{array}{l}\text { Autonomous } \\
\text { Vehicle }\end{array}$ & 5 & 5 & 4 & -11 & 7 \\
\hline
\end{tabular}

TABLE 5: SCENARIOS AND WEIGHTS

\begin{tabular}{|l|l|l|l|l|l|}
\hline & \multicolumn{2}{|l|}{ Benefit Attributes } & Cost Attributes \\
\hline So Scenario & 0.2 & 0.2 & 0.2 & Availability & Cost \\
\hline Scenario 1 & 80 & 50 & 80 & 0.2 & 0.2 \\
\hline Scenario 2 & 80 & 50 & 70 & 70 & 90 \\
\hline Scenario 3 & 80 & 50 & 60 & 60 & 80 \\
\hline Scenario 4 & 80 & 50 & 80 & 40 & 70 \\
\hline Scenario 5 & 80 & 50 & 70 & 80 & 80 \\
\hline Scenario 6 & 80 & 50 & 60 & 70 & 70 \\
\hline Scenario 7 & 80 & 50 & 80 & 50 & 60 \\
\hline Scenario 8 & 80 & 50 & 70 & 80 & 70 \\
\hline Scenario 9 & 80 & 50 & 50 & 70 & 60 \\
\hline
\end{tabular}

TABLE 6: RELATIVE DISTANCE MATRIX FOR EACH SCENARIO AND RANK FOR EACH ALTERNATIVES

\begin{tabular}{|c|c|c|c|c|c|c|c|}
\hline & & \multicolumn{6}{|c|}{ Candidate Technologies } \\
\hline & & \multirow{2}{*}{$\begin{array}{l}\text { V2V } \\
\text { Score }\end{array}$} & \multicolumn{3}{|c|}{ V2I } & \multicolumn{2}{|c|}{ Autonomous Vehicle } \\
\hline & & & Rank & Score & Rank & Score & Rank \\
\hline No Scenario & & 0.883 & 1 & 0.576 & 2 & 0.119 & 3 \\
\hline Scenario 1 & Government sponsored R\&D $\backslash$ Short Term & 0.923 & 1 & 0.584 & 2 & 0.119 & 3 \\
\hline Scenario 2 & Government sponsored R\&D $\backslash$ Medium Term & 0.915 & 1 & 0.564 & 2 & 0.133 & 3 \\
\hline Scenario 3 & Government sponsored R\&D \Long Term & 0.901 & 1 & 0.536 & 2 & 0.156 & 3 \\
\hline Scenario 4 & Tax credit for companies to invest in $\mathrm{R} \& \mathrm{D} \backslash$ Short Term & 0.923 & 1 & 0.573 & 2 & 0.119 & 3 \\
\hline Scenario 5 & Tax credit for companies to invest in $\mathrm{R} \& \mathrm{D} \backslash$ Medium Term & 0.915 & 1 & 0.552 & 2 & 0.132 & 3 \\
\hline Scenario 6 & Tax credit for companies to invest in $\mathrm{R} \& \mathrm{D} \backslash$ Long Term & 0.900 & 1 & 0.522 & 2 & 0.158 & 3 \\
\hline Scenario 7 & Tax credit and rebates for consumers $\backslash$ Short Term & 0.920 & 1 & 0.563 & 2 & 0.124 & 3 \\
\hline Scenario 8 & Tax credit and rebates for consumers $\backslash$ Medium Term & 0.912 & 1 & 0.540 & 2 & 0.138 & 3 \\
\hline Scenario 9 & Tax credit and rebates for consumers $\backslash$ Long Term & 0.899 & 1 & 0.499 & 2 & 0.159 & 3 \\
\hline
\end{tabular}

